\title{
Sub-diffraction limit laser ablation via multiple exposures using a digital micromirror device
}

\author{
Daniel J Heath*, James A Grant-Jacob, Matthias Feinaeugle, Ben \\ Mills, and Robert W Eason \\ Optoelectronics Research Centre, University of Southampton, Southampton, SO17 1BJ, UK \\ *Corresponding author: djh2v07@soton.ac.uk \\ Received XX Month XXXX
}

\begin{abstract}
We present the use of digital micromirror devices as variable illumination masks for pitch-splitting multiple exposures to laser machine the surfaces of materials. Ultrafast laser pulses of length $150 \mathrm{fs}$ and $800 \mathrm{~nm}$ central wavelength were used for the sequential machining of contiguous patterns on the surface of samples in order to build up complex structures with sub-diffraction limit features. Machined patterns of tens to hundreds of microns in lateral dimensions with feature separations as low as $\mathbf{2 7 0} \mathbf{~ n m}$ were produced in electroless nickel on an optical setup diffraction-limited to $727 \mathrm{~nm}$, showing a reduction factor below the Abbe diffraction limit of $\sim 2.7 \mathrm{x}$. This was compared to similar patterns in a photoresist optimised for two-photon absorption, which showed a reduction factor of only $2 x$, demonstrating that multiple exposures via ablation can produce a greater resolution enhancement than via two-photon polymerisation.
\end{abstract}

OCIS codes: (140.3300) Laser beam shaping, (230.6120) Spatial light modulators, (220.4000) Microstructure fabrication, (190.4180) Multiphoton processes, (220.1140) Alignment.

http://dx.doi.org/10.1364/AO.99.099999

\section{Introduction}

The increase in feature density achievable via laser machining is an ever-present goal in optics. The well-known Abbe diffraction limit, $R=\lambda /(2 \mathrm{NA})$, defines the finest resolution, $R$, feasible in a single optical projection, where $\lambda$ is wavelength and NA is the numerical aperture of the imaging system. The use of shorter wavelengths has achieved much progress, with $13.5 \mathrm{~nm}$ wavelength EUV techniques able to machine structures on the $\sim 10 \mathrm{~nm}$ scale [1]. These technologies require short wavelength lenses, or all-reflective optical systems for EUV lithography; multiple material improvements and image-formation methods have also aided the attainment of increasingly fine resolution produced by optical techniques [2].

Alternative techniques increase final feature density without addressing the factors of the Abbe diffraction limit. Double patterning [3-5], also often referred to as multiple exposures [6,7], are one such class of techniques. In this work, a given intensity mask to produce a final desired pattern is broken down into several masks of reduced pitch to be exposed sequentially, which will be referred to as pitchsplitting multiple exposures (PSME). A spatial light modulator, namely a digital micromirror device (DMD), is used to rapidly switch between these different masks between femtosecond pulse exposures. Further, the potential of PSME when applied to the ablation of metal is compared to the well-reported exposure of photo-resist. Via ablation in nickel, a reduction factor of $\sim 2.7 \mathrm{x}$ in final feature spacing as compared to the Abbe diffraction limit is demonstrated, while a factor of $2 \mathrm{x}$ is shown via polymerisation in photo-resist. The reduction factor of $\sim 2.7 \mathrm{x}$ is observed in nickel when the process is performed on two different optical systems with numerical apertures of 0.55 and 0.40 , indicating that this may be a material-dependent limit.

\section{Experimental setup}

The DMDs used in this work were Texas Instruments DLP3000 [8] and DLP7000 [9]. The DLP3000 consists of an array of 608 by 684 individually controllable, $7.6 \mu \mathrm{m}$ wide mirrors, arranged in a diagonal-square lattice such that the display aspect ratio is 854:480. The centre-to-centre distance between adjacent mirrors in the horizontal and vertical planes was $10.8 \mu \mathrm{m}$. The direction of the diffraction order of maximum intensity observed from a DMD surface will depend on the direction of mirror tilt, which in this case was along the diagonal of each square mirror. The DLP7000 surface was thus rotated by $45^{\circ}$ about the axis of the chosen 
diffraction order (described below), to maintain the direction of the beam line to be parallel to the optical bench. The DLP7000 consists of an array of 768 by 1024 individually controllable, $13.7 \mu \mathrm{m}$ wide mirrors, arranged in a square lattice. The centre-to-centre distance between adjacent mirrors in the horizontal and vertical planes was then 19.3 $\mu \mathrm{m}$. With angular orientations relative to the plane of both DMD surfaces of either $+12^{\circ}$ or $-12^{\circ}$, mirrors will be described as being 'on' or 'off', respectively.

As a result of the periodic structure of a DMD, when working with a spatially coherent light source, multiple diffraction peaks are produced. The output angles of these peaks can be determined from the grating equation for nonnormal incidence, $\sin \left(\theta_{\mathrm{i}}\right)-\sin \left(\theta_{\mathrm{m}}\right)=\mathrm{m} \lambda / \mathrm{d}$, where $\mathrm{m}$ is the order of diffraction, $\lambda$ is the laser wavelength, $d$ is the mirror spacing, and $\theta_{\mathrm{i}}$ and $\theta_{\mathrm{m}}$ are the incident and diffracted angles. In this case, the DMD acted as a blazed grating, which was taken advantage of in this work in order to increase the percentage of diffracted light present in a single chosen diffraction order to $\sim 30 \%$.

A Ti:sapphire amplifier was used to produce ultrashort laser pulses (150 fs, $800 \mathrm{~nm}$ central wavelength) with a maximum single pulse energy of $2 \mathrm{~mJ}$. Periods between exposures were typically $\sim 50 \mathrm{~ms}$. Primarily, a femtosecond source was chosen for the strong multi-photon absorption effects, which are required for sub-diffraction-limit multiple exposures in photo-resists [7]. Two similar experimental setups were used in order to determine the reduction factor limit to resolution produced with PSME via ablation, with a generic form shown in Fig. 1. The details of the setup optimised for a $50 \mathrm{x}$ or $100 \mathrm{x}$ objective lens, used for both ablation and two-photon polymerisation (2PP), has been described previously [10]. The only significant differences between this line, which used a DLP3000 DMD, and the second setup were the use of a TOPAG Gaussian-to-Top Hat beam shaping lens, DLP7000 DMD chip and 20x objective lens for large-area machining. The numerical apertures for the $100 \mathrm{x}, 50 \mathrm{x}$ and $20 \mathrm{x}$ objectives were $0.55,0.42$ and 0.40 respectively, and thus the calculated resolution limits, $R$, were $727 \mathrm{~nm}, 952 \mathrm{~nm}$ and $1.00 \mu \mathrm{m}$.

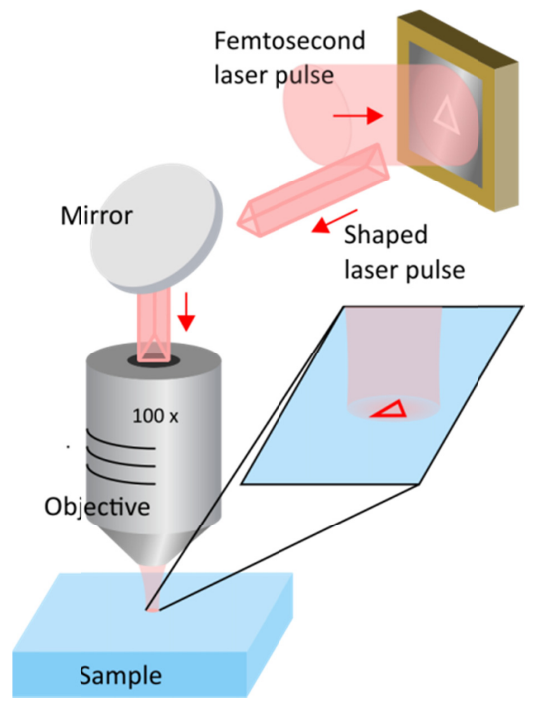

Fig. 1 Simplified schematic of the experimental setup. A circular intensity top-hat intensity distribution is incident on the DMD, which displays an arbitrary mask (in this case, a triangle). The output shaped intensity distribution is directed to an objective lens towards a sample, where the displayed mask is imaged and the material response correlates with the spatial distribution displayed on the DMD.

\section{Pitch-splitting multiple exposures}

While diffraction limits the resolution of a structure machined via a single projected intensity profile, a second, different intensity profile projected at a later time can be used to modify the structure to produce a final pattern that would be impossible to create in a single exposure. The principle of PSME is the following: a desired pattern is split into $m$ intensity masks, where each mask's pitch (when projected) is approximately $m$ times larger than that of the final desired pattern. As PSME requires multiple intensity masks, and for particularly small periods of feature separation small shifts of an overall intensity pattern are needed, there is a requirement for accurate positioning of a mask image at the sample compared to the positions of previous images of masks. This concern in multiple exposures, referred to as Critical Dimension (CD) Uniformity is discussed in depth by Hazelton et al. [4].

The above problem of CD Uniformity control is addressed here using a spatial light modulator, such as a DMD, as the intensity mask. The DMD masks are updatable digitally in a millisecond timescale [9], with the DMD itself requiring no repositioning. Dynamic, digital intensity mask display is inherently faster than mechanically replacing intensity masks, and there is no risk of misalignment between previous and future projected intensity patterns. This paper describes results from the use of a DMD, acting as a dynamic intensity mask for PSME via ablation and two-photon polymerisation, with minimum final resolution of $270 \mathrm{~nm}$, achieved on an experimental setup diffraction-limited to a theoretical value of $727 \mathrm{~nm}$. The resolution reduction factor of 2 associated with two-photon resists has been surpassed via ablation, with a consistent reduction factor of $\sim 2.7 \mathrm{x}$ encountered when ablating nickel with two different optical setups with different numerical apertures (NA $=0.40$ and 0.55). DMDenabled ablation and multi-photon polymerisation have been demonstrated previously [11-13], and the method described here is expected to enhance achievable resolution for PSME.

\section{A. PSME via two-photon polymerisation}

To predict limits to the reduction of final feature resolution possible via PSME, it is important to note that the final machined feature's structure depends on material response to exposed intensity distributions, rather than simply the resolution of the intensity distribution itself. Such a limit was proposed by Ooki et al. [6,7] for PSME in a two-photon resist when using a sinusoidal intensity pattern shifted by half a period, with at most a halving of the period of the final material response being possible. A similar two-photon resist, Nanoscribe IPG-780 [14], was used in this work, and Ooki et al's argument can be extended to show the same factor of 2 reduction limit for arbitrary 2D intensity distributions shifted laterally by any distance as will be described below.

The density of a photo-initiator chemical, J, in a two-photon resist, with initial density $J_{0}$, corresponds to the degree of polymerisation achieved (with $J=0$ corresponding to complete polymerisation). The initiator's rate of decrease 
with respect to time, $t$, is proportional to the square of the incident intensity for a two-photon resist [15]. By integrating w.r.t. time, this means that after 1 exposure to intensity $I_{1}$ the new initiator density $J_{1}$ is given by Equation 1 .

$$
J_{1}(t)=J_{0} \exp \left(-c_{2 P} I_{1}^{2} t\right)
$$

Here, $c_{2} \mathrm{P}$ is a constant of integration, and the intensity was assumed constant during the exposure time, though this was purely to simplify the expression. The squared intensity-time product would be replaced by a squared intensity integral with respect to time in a more detailed analysis, but this would not alter the order of the polynomial dependence on intensity, and hence the possible resolution reduction factor, as will be explained below. Explicit dependence on lateral dimensions $x$ and $y$ have been omitted from initiator densities and intensities, to ease notation. If the exposure time for the $n$th exposure is $T_{n}$, with intensity distribution $I_{n}$, then the initiator density after $N$ exposures is given by Equation 2 .

$J_{N}\left(T_{N}\right)=J_{N-1} \exp \left(-c_{2 P} I_{N}^{2} T_{N}\right)=J_{0} \exp \left(-c_{2 P} \sum_{n=1}^{N} I_{n}^{2} T_{n}\right)$

The final photo-initiator density is thus in response to an effective squared intensity-time product given by the summation in Equation 2. The upper limit to the spatial frequencies present in the final structure produced via 2PP can be inferred from the spatial Fourier transform of this summation. An intensity pattern containing a maximum spatial frequency of $k_{\max }$ may produce a material response in two-photon resist proportional to the square of the intensity pattern, hence the maximum spatial frequency present in this response is then $2 k_{\max }$. As the Fourier transform is distributive, the number of exposures $N$ does not affect this limit; the maximum frequency present in the transform of the sum of multiple functions cannot be greater than the maximum frequency present in the transforms of the functions individually. This is key to understanding why the same limits to resolution reduction factor are seen when differing numbers of exposures are used in Section 4.A.

While 2PP has been used to produce structures with resolutions below this limit, additional methods to alter the material response must be used [15]. One such technique relies on the diffusion of a "quenching" molecule and a slow laser scanning speed [16], which effectively decays the material response over time in regions not completely polymerised, such that $2 \mathrm{PP}$ is closer to a 'threshold' process, as discussed in the next section.

\section{B. PSME via ablation}

The material response to incident intensity as a result of ablation is a different mathematical form to that of 2PP. As a simple model, assume that the depth profile after exposure $n$, $D_{n}$, is given by Equation 3 .

$$
D_{n}=D_{n-1}+\Delta D_{n}
$$

Where explicit dependencies on lateral coordinates have again been dropped for brevity. Here, $\Delta D_{n}$ is the change in depth caused by exposure $n$, and $D_{n-1}$ is the depth profile after the previous exposure. Ablation is often considered a thresholded process $[17,18]$, and so to a first order approximation ablation may be assumed to only occur above some threshold intensity $I_{t h}$. While this is not strictly true, and some polynomial reflecting a sharp, but finite gradient rise in material removal probability around the threshold intensity would be more accurate, a true threshold approximation serves to illustrate the possibility of enhanced resolution using PSME via ablation. $\Delta D_{n}$ may then take the first order approximate form in Equation 4, where the change in depth profile is proportional to intensity above threshold.

$$
\Delta D_{n} \propto\left\{\begin{array}{cl}
\left(I_{n}-I_{t h}\right) & \text { Where } I_{n}>I_{t h} \\
0 & \text { Otherwise }
\end{array}\right.
$$

Next consider, the lateral spatial frequency components present in $\Delta D_{n}(x, y)$ to determine the maximum possible spatial frequency in an ablated structure, $D_{n}$, produced via PSME. Here, the sharp cut-off between affected and unaffected regions shown in Equation 4 would imply infinitely high spatial frequencies must be present, allowing a final structure to have arbitrarily high resolution. Of course, such a sharp cut-off is only an approximation, and the model is presented merely to demonstrate the impact of material response on final possible resolutions; the reduction factor limit in PSME via ablation is not necessarily limited to the same value as via 2PP. A more complete modelling of ablation depth with successive femtosecond exposures has been proposed by others $[17,18]$, wherein a threshold is considered an acceptable approximation. It is possible that different materials would yield different nonlinearities in their responses to incident intensity, i.e. sharper ablation thresholds, and could return greater reductions in the resolution limit.

Note that, while Equations (1) and (4) imply spatial frequencies above the Abbe diffraction limit may be present in the material response after a single exposure, it does not allow for the machining of patterns with individual feature spacing below this limit in a single exposure; to achieve this, multiple exposures must be made. A 1D example is given in Fig. 2, for the ablation case; special attention is given to this mechanism as low feature width structuring may only emerge after several exposures, while low feature width structures are typically produced in each exposure during 2PP, and has been described by others [15]. A Gaussian intensity (solid red line) and machined depth profile (solid black line after exposure, dashed blue line before exposure) are plotted on the same axes (arbitrary units), showing the material response (depth of ablation) before and after 1-9 exposures of mutually shifted Gaussian intensities. The threshold intensity level, $I_{t h}$, is shown by a dashed cyan line. 

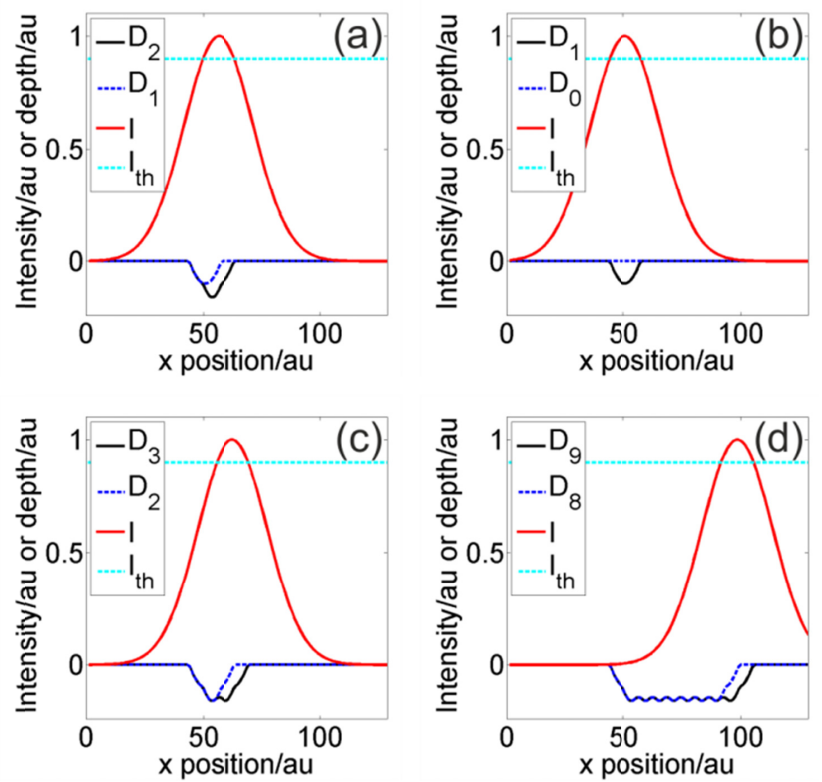

Fig. 2 A schematic of PSME for ablation, where depth profile $D_{n}$ modification only occurs as a result of intensity $I$ above threshold $I_{t h}$. The position of Gaussian intensity profiles and the depth profiles before and after (a) One exposure (b) Two exposures (c) Three exposures and (d) Nine exposures are shown.

Fig. 2(a) shows the depth of ablation predicted by Equation 3 when only a small central region of a Gaussian intensity profile is above the ablation threshold. Fig. 2(b) shows additional depth ablated by a subsequent pulse at a position a small distance away from the initial exposure position; note that at this point no periodic structuring is clear in the overall depth profile. After 3 exposures in Fig. 2(c), two adjacent small depth modulations are clear at the bottom of an overall deeper, wider hole. After 9 exposures, as in Fig. 2(d), a structure with period equal to the successive intensity shift distance has been produced, at the bottom of an overall wider, deeper ablated hole. The number of exposures required before periodic structures emerge varies with incident distribution shape, the size of successive shifts, and the threshold of ablation, even in this simple 1D model, but typically increases as the desired feature separation decreases.

\section{Experimental results}

In this section, results from the exposure of nickel substrates and two-photon resists are shown. The intensity masks used, take the form of binary patterns displayed on the DMDs, and these masks are either translated or rotated on the DMDs to perform PSME. The results are shown to agree with the models presented in Section 3.A and 3.B, and the resolution reduction factors achieved via ablation and $2 \mathrm{PP}$ are compared.

\section{A. PSME via ablation}

Initially, spatial intensity patterns in the form of gratings (as in previous work [10]) were projected onto samples. The machined material was an electroless nickel coating $(\sim 5 \mu \mathrm{m}$ thick), which has an amorphous structure, on copper, and was chosen to minimise problems resulting from any material irregularities due to grain boundaries [19]. Typical ablation depths per pulse were interferometrically measured to be $\sim 10 \mathrm{~nm}$ at the highest fluences used on electroless nickel, with a maximum total ablation depth of $\sim 600 \mathrm{~nm}$ after $\sim 50$ exposures, well below the thickness of the electroless nickel coating. An example pattern as displayed on the DMD is shown in the inset of Fig. 3(a), which comprised of alternating lines of 7 'on' pixels and 17 'off' pixels. This 'on' line width was close to the minimum viable for imaging without significant diffractive losses on the different setups, and was used to produce the finest possible features in each exposure. These line widths were used for all grating DMD masks used, and mutual shifts were limited to factors of 24 for even spacing between exposures (i.e. the overall pattern would be shifted by $2,3,4,6,8$ or 12 pixels vertically between exposures, for $12,8,6,4,3$ or 2 total exposures respectively). A final predicted depth profile, using a 2D form of the theory from Fig. 2, is shown in Fig. 3(b) after 4 exposures, where black corresponds to zero depth. The grating patterns for this predicted profile were shifted vertically by 6 pixels between each exposure.

Final ablated patterns on the sample were formed using single pulse exposures for each translated mask, where the same grating pattern would be translated downwards on the DMD after each exposure, to interleave machined horizontal lines. Resulting ablated patterns produced on the DLP7000 line are shown in scanning electron microscope (SEM) images in Fig. 3(c), (d) and (e), with matching resolutions between theoretical and experimental values shown in (f). Blue line profiles reflecting the horizontally summed greyscale values of the SEM images are shown in Fig. 3(d) and (e), to highlight the periodicity of the structure. 

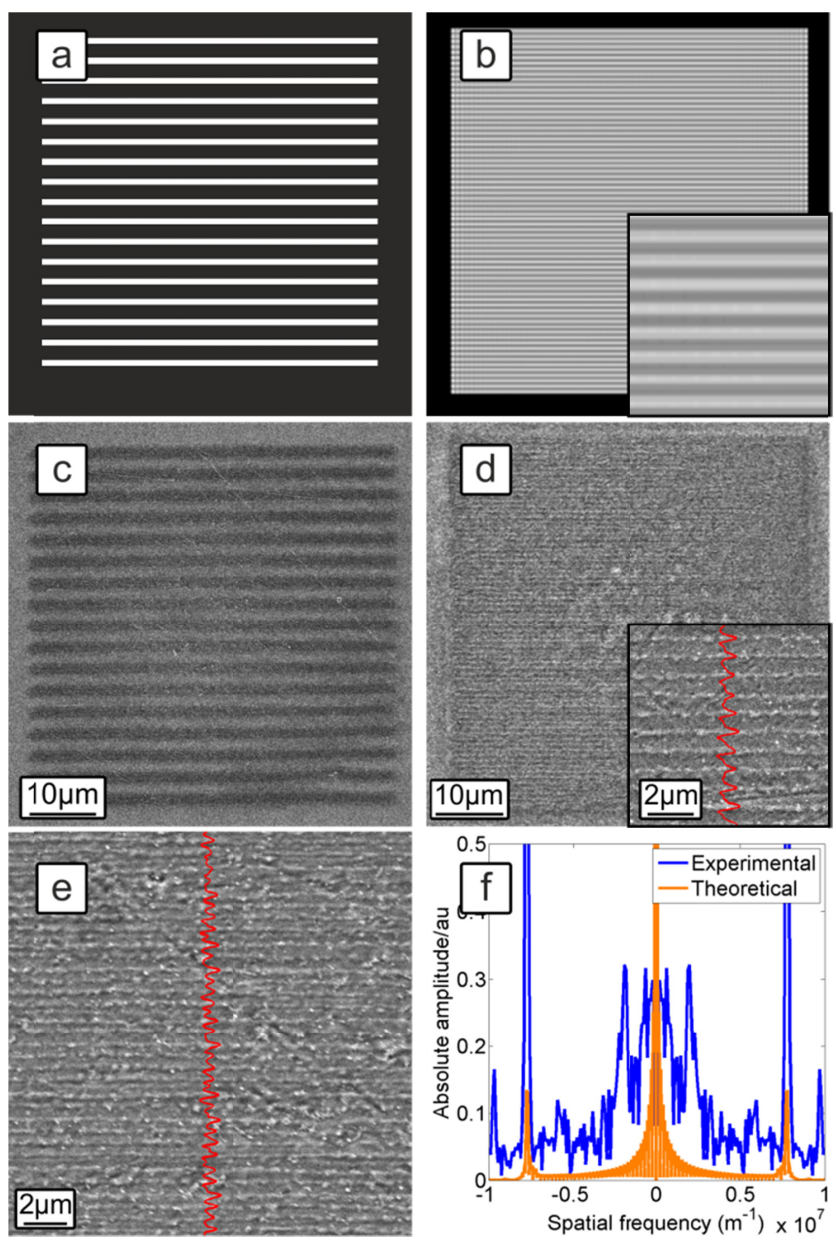

Fig. 3 Interleaved exposures of 2D grating patterns. (a) the grating image as displayed on the DMD, (b) simulated depth profile after 4 PSME, with the inset showing a higher magnification region. SEM images of the resultant structures after exposure on electroless nickel are shown in (c) after 1 exposure, (d) after 4 exposures with the inset showing a higher SEM magnification image (e) after 6 exposures. Each exposure was at a fluence of $38 \mathrm{~mJ} / \mathrm{cm}^{2}$ with the $20 \mathrm{x}$ objective, (f) the FFT of horizontally summed greyscale values in the theoretical depth profile from (b) and the experimental profile from (d).

The ablated structure after a single exposure of the grating image in Fig. 3(a) produced lines with a period of $3.28 \mu \mathrm{m}$ $\pm 20 \mathrm{~nm}$ in Fig. 3(c). This period was measured both by averaging the separation between machined lines directly, and by taking the Fourier transform (FT) of the SEM image, ensuring that a peak in the frequency spectrum corresponding to the averaged measurement was present. Errors are estimated by assuming a \pm 0.5 pixel error in the scale bar automatically recorded on SEM images, which were used to provide a scale for the FTs. The SEM image in Fig. 3 (d) shows the ablated structure after 4 exposures, with the DMD image shifted downwards by 6 pixels between exposures, resulting in lines with a period of $815 \mathrm{~nm} \pm 5 \mathrm{~nm}$. The inset shows a higher SEM magnification, with a blue profile highlighting the structure - produced by taking a horizontal average of the greyscale pixel values in the image. The SEM image in Fig. 3(e) shows the structure produced after 6 exposures with mutual image shifts of 4 pixels, and a resultant period of $546 \mathrm{~nm} \pm 2 \mathrm{~nm}$. The final periods seen in Fig. 3(d) and (e) of $815 \mathrm{~nm}$ and $546 \mathrm{~nm}$ correspond well to the expected reduction in pitch from the $3.28 \mu \mathrm{m}$ initial pitch in (c) for PSME of 4 and 6 exposures respectively. Fig. 3(f) shows the Fourier transforms (FTs) of the horizontally summed greyscale values from (b) and (d), to compare the predicted vs. experimental obtained final feature resolutions. The size scale assumed for (b) is inferred from a mapping of (a) onto the SEM image in (c). Each exposure was made at a fluence of $\sim 38 \mathrm{~mJ} / \mathrm{cm}^{2}$, close to the ablation threshold of nickel [20].

In order to test the limits of the technique for the production of more complex patterns by simultaneously attempting to achieve a near-continuous range of final resolutions, Archimedean spirals rotated relative to each other (described in polar coordinates by equations of the form $r=a+b \theta^{c}$, with $a=0$ and $c>1$ in our case, such that the radial distance between 'turns' of the spiral decrease towards the centre) were displayed on the DMD in both setups. Fig. 4(a) shows the relative rotated positions of eight sequential spirals as an example, while the inset shows one such spiral as displayed on the DMD. Final resolutions hence vary from high at the centre of the structures, to low at the edges, in the case of exposing rotated Archimedean spirals. Stated period results are measured by taking the average of 3-4 adjacent machined lines, over which the period is approximately uniform.
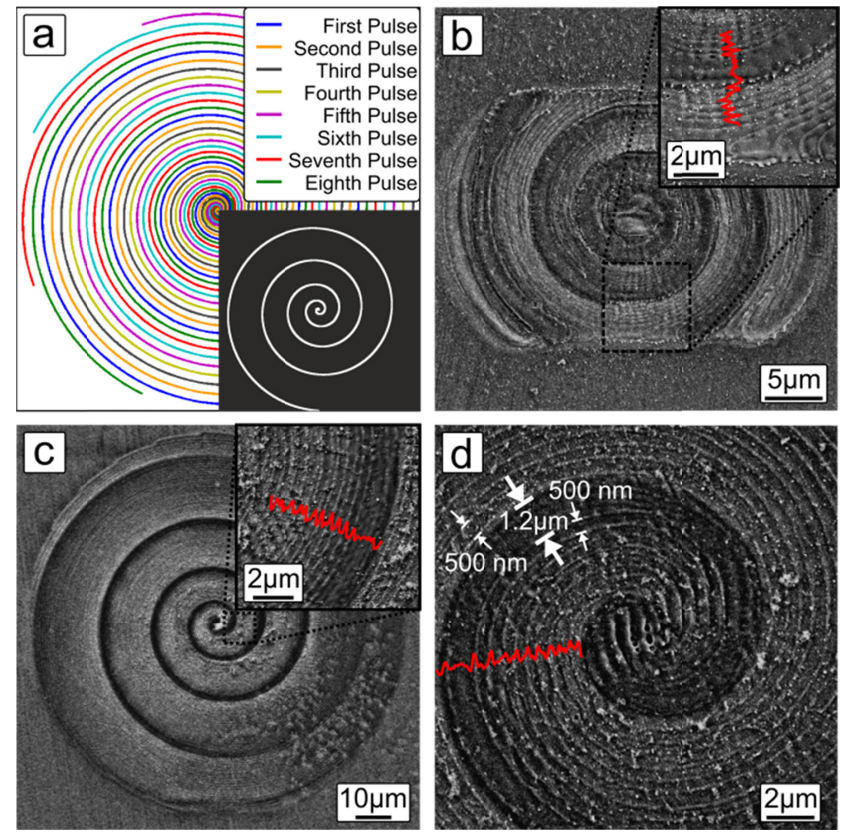

Fig. 4 SEM images of Archimedean spirals ablated in electroless nickel. (a) Relative positioning for eight sequentially rotated spirals, inset shows a single spiral DMD mask. (b) Produced with the 100x objective on the DLP3000 line, machined in 13 pulses at $4.70 \mathrm{~J} / \mathrm{cm}^{2}$, inset shows a higher magnification SEM image. (c) Produced in 25 pulses at $38 \mathrm{~mJ} / \mathrm{cm}^{2}$, with the $20 \mathrm{x}$ objective on the DLP7000 line. The inset shows a higher magnification SEM image. (d) Shows the central region of a structure produced in 10 pulses at 38 $\mathrm{mJ} / \mathrm{cm}^{2}$ with the $20 \mathrm{x}$ objective. 
Fig. 4(b) shows a resulting final structure machined using the $100 x$ objective, with the inset displaying a higher SEM magnification image, produced in 13 pulses at $4.70 \mathrm{~J} / \mathrm{cm}^{2}$. The structure appears cropped in the vertical, as the DLP3000 chip has an 854:480 aspect ratio display, and was not able to display the entire spiral as in the Fig. 4(a) inset. As the structures contain a near-continuous range of feature separations by design, peaks in the 2D FT corresponding to the minimum achieved period were not necessarily high and sharp compared to background, and were hence not conclusive alone. Instead, periods stated were measured directly from the SEM images by averaging the spacing of corrugations over a line perpendicular to the direction of turns of the spirals, and repeating this measurement at several nearby regions, before being verified via the presence of a peak in the 2D FT. The finest period of $270 \mathrm{~nm} \pm 2 \mathrm{~nm}$ was achieved near the centre of the structure in Fig. 4(b), with a period of $\sim 350 \mathrm{~nm}$ seen nearer to the edge. The factor of $\sim 100 \mathrm{x}$ fluence difference used to generate the structure in Fig. 4(b) compared to (c) and (d) demonstrate that the technique is viable over a wide intensity range, with ablation depths (in the $<100 \mathrm{~nm}$ range, as stated earlier) consistent with those found in nickel and its alloys [20,21].

Figure 4(c) shows a similar structure machined with the $20 \mathrm{x}$ objective, which was produced in 25 pulses at $38 \mathrm{~mJ} / \mathrm{cm}^{2}$. The finest resolution of ridges in the structure was $360 \mathrm{~nm}$ $\pm 2 \mathrm{~nm}$, shown at the centre of the inset. Fig. 4(d) shows the central region of a spiral produced with 10 pulses at 38 $\mathrm{mJ} / \mathrm{cm}^{2}$ with the $20 \mathrm{x}$ objective, demonstrating $\sim 500 \mathrm{~nm}$ resolution features almost to the very centre of the structure. Visible in (b), (c) and highlighted with white arrows in (d) are broad spirals, surrounded by regions which appear to have high periodicity - e.g. a $1.2 \mu \mathrm{m}$ linewidth spiral of little depth modulation with $\sim 500 \mathrm{~nm}$ linewidth spiral features to either side, as indicated in Fig. 4(d). These broad curves correspond to the edges of the overall wide, deep hole structure predicted in Fig. 2. Also apparent is a build-up of 'nanofoam', which has been previously investigated [22], and is progressively less concentrated towards regions patterned with later pulses in the series of machining exposures. Such nanofoam is typical of multi-exposure femtosecond ablation machining.. At the centre of the spiral, visible in (d), laserinduced periodic surface structuring (LIPSS) [23] occurred as a result of the rotating pattern in the central region being exposed to all pulses, and repeated material exposure to femtosecond pulses often results in LIPSS, though neither this nor the nanofoam were investigated further during this study, and are mentioned here merely to explain the presence of these features.

With a diffraction limit of $727 \mathrm{~nm}$ and final feature resolution of $\sim 270 \mathrm{~nm}$ with the $100 \mathrm{x}$ objective, and a limit of $1.00 \mu \mathrm{m}$ and final feature resolution of $\sim 360 \mathrm{~nm}$ with the $20 \mathrm{x}$ objective, resolutions approximately $2.7 \mathrm{x}$ smaller than the diffraction limit were produced by PSME in both cases. The similarity of the reduction factor seen for PSME via ablation in nickel on different DMDs, with different numerical apertures, is the primary reason for including the result obtained using the 100x objective seen in Fig. 4(b). While it was shown in section 3.A and confirmed experimentally in section 4.B that a two-photon resist generally leads to a resolution reduction factor limit of 2 on whichever optical system is used for the two-photon process, the reduction factor limit for PSME via ultrafast ablation in nickel appears to be $\sim 2.7 \mathrm{x}$, applicable when using different optical systems.

\section{B. PSME via photo-polymerisation}

In order to compare PSME via ablation using DMD image translation to PSME via 2PP, a resist optimised for $2 \mathrm{PP}$ (Nanoscribe IP-G 780) was used [14]. Only the 50x and 100x objectives were capable of producing the high intensities needed for two-photon polymerisation. The results in Fig. 5 show a $\sim 10 \mu \mathrm{m}$ thick structure produced in 13 shots at 3.0 $\mathrm{J} / \mathrm{cm}^{2}$ using the $50 \mathrm{x}$ objective, which was limited to $952 \mathrm{~nm}$ resolution in a single exposure, with a final minimum lateral resolution of $481 \mathrm{~nm} \pm 4 \mathrm{~nm}$, which was equal to the width of single lines produced during each pulse. The high number of exposures at the central region resulted in damage to the structure. Incomplete polymerisation was observed at 2.4 $\mathrm{J} / \mathrm{cm}^{2}$, with polymerisation fluences in this range being typical for similar experimental conditions [24].

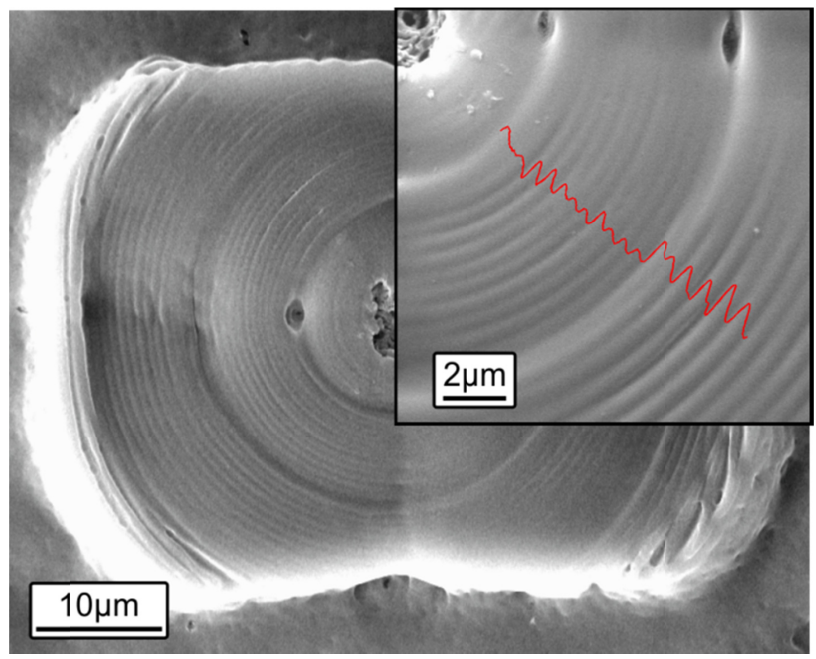

Fig. 5 SEM image of an Archimedean spiral photopolymerised in IP-G 780 photoresist, produced with the $50 \mathrm{x}$ objective. The structure was fabricated using 13 pulses, 3.0 $\mathrm{J} / \mathrm{cm}^{2}$, while the inset shows a higher SEM magnification, with a finest resolution of $480 \mathrm{~nm}$.

A summary of the results achieved with PSME via DMD mask translation is given below in Table 1 . For the case of 2PP, the enhancement of resolution beyond the Abbe diffraction limit was approximately a factor of 1.98 , close to the limit of 2 expected with a two-photon resist [7]. The overlapping ablation method introduced in section 4.A, however, surpasses this with an enhancement factor of $2.7 \mathrm{x}$. The final structures shown, produced via ablation on different experimental setups, achieved the minimum resolution in differing numbers of pulses. However, as explained in Section 3.A, the number of exposures used is not of fundamental importance to the technique; rather, it is the interaction between material response, the pitch of patterns machined in each exposure, and relative shifts between exposures that determines the number of exposures required to reach a final pitch.

Table 1. A summary of the results obtained via exposures of rotated spirals 


\begin{tabular}{l|l|l|l}
\hline & \multicolumn{2}{|c}{ Objective Used } \\
\hline & \multicolumn{2}{|c}{$\mathbf{2 0 x} \mathbf{5 0 x} \mathbf{1 0 0 x}$} \\
\hline Resolution limit & $1.00 \mu \mathrm{m}$ & $952 \mathrm{~nm}$ & $727 \mathrm{~nm}$ \\
\hline Material & $\begin{array}{l}\text { Electroless } \\
\text { nickel }\end{array}$ & IP-G 780 & $\begin{array}{l}\text { Electroless } \\
\text { nickel }\end{array}$ \\
\hline $\begin{array}{l}\text { Resolution } \\
\text { produced }\end{array}$ & $364 \mathrm{~nm}$ & $481 \mathrm{~nm}$ & $267 \mathrm{~nm}$ \\
\hline $\begin{array}{l}\text { Number of } \\
\text { exposures }\end{array}$ & 25 & 13 & 13 \\
\hline $\begin{array}{l}\text { Resolution } \\
\text { reduction factor }\end{array}$ & 2.75 & 1.98 & 2.73 \\
\hline Process & Ablation & $2 \mathrm{PP}$ & Ablation \\
\hline
\end{tabular}

patterning requirements for optical lithography and prospects for optical extension without double patterning," J. Micro/Nanolithography, MEMS MOEMS 8(1),11003 (2009).

5. T. O. Masato Shibuya Masaya Komatsu and H. Ooki, "Performance of resolution enhancement technique using both multiple exposure and nonlinear resist," Jpn. J. Appl. Phys. MS178,864-867 (1994).

6. H. Ooki, D. P. Coon, S. Owa, T. Sei, and K. Okamoto, "Experimental study on nonlinear multiple-exposure method," in Microlithography'97, G. E. Fuller, ed. (1997), pp. 85-93.

7. H. Ooki, K. Masaya, and S. Masato, "A Novel SuperResolution Technique for Optical LithographyNonlinear Multiple Exposure Method," Jpn. J. Appl. Phys. 33.2A,L177-L179 (1994).

\section{Conclusion}

A DMD has been used for the PSME via ablation of features up to $\sim 2.7 \mathrm{x}$ below the diffraction limit of two experimental setups. Material response has been shown to be key to understanding the difference in final feature resolution, and future work will focus on determining the mechanism of this material response to identify materials with different PSME via ablation resolution reduction factors. DMD intensity mask translation for PSME has been used to rapidly and precisely align successive masks with $\sim 100 \mathrm{~nm}$ lateral translations per pixel shift on the DMDs, potentially allowing overall structures to be machined over a few millisecond timescale. PSME is an important tool in the ongoing miniaturisation of laser-machined patterns. We have extended the application of DMDs for laser machining by demonstrating their relevance to PSME, providing a rapid and low-cost alternative to the production and alignment of sequential intensity masks, as well as extending the application of PSME to ablation.

Acknowledgement. The authors are grateful to the Engineering and Physical Sciences Research Council (EPSRC) under grant numbers EP/L022230/1, EP/J008052/1 and EP/N03368X/1.

The data for this work is accessible through the University of Southampton Institutional Research Repository DOI:10.5258/SOTON/390704.

\section{References}

1. R. Peeters, S. Lok, J. Mallman, M. van Noordenburg, N. Harned, P. Kuerz, M. Lowisch, E. van Setten, G. Schiffelers, A. Pirati, J. Stoeldraijer, D. Brandt, N. Farrar, I. Fomenkov, H. Boom, H. Meiling, and R. Kool, "EUV lithography: NXE platform performance overview," SPIE Adv. Lithogr. 9048(February 2013),90481J (2014).

2. T. Ito and S. Okazaki, "Pushing the limits of lithography," Nature 406(6799),1027-31 (2000).

3. K. G. Kemp, "Lithographic method using double exposure techniques, mask position shifting and light phase shifting, US Patent No 5308741," U.S. patent 5308741 (1994).

4. A. J. Hazelton, S. Wakamoto, S. Hirukawa, M. McCallum, N. Magome, J. Ishikawa, C. Lapeyre, I. Guilmeau, S. Barnola, and S. Gaugiran, "Double-

8. Texas Instruments, "DLP 3000," http://www.ti.com/product/dlp3000\#parametrics.

9. Texas Instruments, "DLP7000," http://www.ti.com/product/dlp7000.

10. D. J. Heath, B. Mills, M. Feinaeugle, and R. W. Eason, "Rapid bespoke laser ablation of variable period grating structures using a digital micromirror device for multi-colored surface images," Appl. Opt. 54(16),4984-4988 (2015).

11. B. Mills, M. Feinaeugle, C. L. Sones, N. Rizvi, and R. W. Eason, "Sub-micron-scale femtosecond laser ablation using a digital micromirror device," J. Micromechanics Microengineering 23(3),35005 (2013).

12. B. Mills, J. A. Grant-Jacob, M. Feinaeugle, and R. W. Eason, "Single-pulse multiphoton polymerization of complex structures using a digital multimirror device," Opt. Express 21(12),14853-14858 (2013).

13. Y.-C. Li, L.-C. Cheng, C.-Y. Chang, C.-H. Lien, P. J. Campagnola, and S.-J. Chen, "Fast multiphoton microfabrication of freeform polymer microstructures by spatiotemporal focusing and patterned excitation.," Opt. Express 20(17),19030-8 (2012).

14. J. Li, J. Mu, B. Wang, W. Ding, J. Liu, H. Guo, W. Li, C. $\mathrm{Gu}$, and $\mathrm{Z}$. Y. Li, "Direct laser writing of symmetrybroken spiral tapers for polarization-insensitive three-dimensional plasmonic focusing," Laser Photonics Rev. 8(4),602-609 (2014).

15. M. Malinauskas, M. Farsari, A. Piskarskas, and S. Juodkazis, "Ultrafast laser nanostructuring of photopolymers: A decade of advances," Phys. Rep. 533(1),1-31 (2013).

16. I. Sakellari, E. Kabouraki, D. Gray, V. Purlys, C. Fotakis, A. Pikulin, N. Bityurin, M. Vamvakaki, and M. Farsari, "Diffusion-Assisted High Resolution Direct Femtosecond Laser Writing," ACS Nano 6(3),120210105238000 (2012).

17. J. R. V. De Aldana, C. Méndez, L. Roso, and P. Moreno, "Propagation of ablation channels with multiple femtosecond laser pulses in dielectrics: numerical simulations and experiments," J. Phys. D. Appl. Phys. 38(16),2764-2768 (2005).

18. J. R. Vázquez de Aldana, C. Méndez, and L. Roso, "Saturation of ablation channels micro-machined in fused silica with many femtosecond laser pulses.," Opt. Express 14(3),1329-38 (2006). 
19. P. T. Mannion, J. Magee, E. Coyne, G. M. O'Connor, and T. J. Glynn, "The effect of damage accumulation behaviour on ablation thresholds and damage morphology in ultrafast laser micro-machining of common metals in air," Appl. Surf. Sci. 233(14),275-287 (2004).

20. M. D. Shirk and P. A. Molian, "A review of ultrashort pulsed laser ablation of materials A review of ultrashort pulsed laser ablation of materials," J. Laser Appl. 10(1),18-28 (1998).

21. J. P. Mcdonald, S. Ma, T. M. Pollock, S. M. Yalisove, J. A. Nees, J. P. Mcdonald, S. Ma, T. M. Pollock, and S. M. Yalisove, "Femtosecond pulsed laser ablation dynamics and ablation morphology of nickel based superalloy CMSX-4 Femtosecond pulsed laser ablation dynamics and ablation morphology of nickel based superalloy CMSX-4," 93111(2008),1-8 (2016).

22. J. A. Grant-Jacob, B. Mills, and R. W. Eason, "Parametric study of the rapid fabrication of glass nanofoam via femtosecond laser irradiation," J. Phys. D. Appl. Phys. 47(5),55105 (2014).

23. E. L. Gurevich and S. V. Gurevich, "Laser Induced Periodic Surface Structures Induced by Surface Plasmons Coupled via Roughness," Appl. Surf. Sci. 302,1-16 (2013).

24. M. Malinauskas, A. Žukauskas, G. Bičkauskaitė, R. Gadonas, and S. Juodkazis, "Mechanisms of threedimensional structuring of photo-polymers by tightly focussed femtosecond laser pulses," Opt. Express 18(10),10209 (2010). 